\title{
Efficient linear estimation problem in the bivariate Kotz distribution under dependence assumptions
}

\author{
R. GUTIÉRREZ-JÁIMEZ*†, J. D. JIMÉNEZ-LÓPEZ† and R. GUTIÉRREZ-SÁNCHEZ§ \\ $\dagger$ †epartamento de Estadística e Investigación Operativa, Universidad de Granada Campus \\ Fuentenueva, s/n, 18071 Granada, Spain \\ ¥Departamento de Estadística e Investigación Operativa, Universidad de Jaén Paraje \\ Las Lagunillas, s/n, 23071 Jaén, Spain \\ §Departamento de Estadística e Investigación Operativa, Universidad de Granada Campus \\ Fuentenueva, s/n, 18071 Granada, Spain
}

(Revised 18 December 2003; in final form 5 October 2004)

\begin{abstract}
Our aim in this article is to obtain efficient estimators of the parameters of the bivariate Kotz type distribution considering a particular matrix-variate joint dependence between the sample random vectors. As the normal law is a particular Kotz type distribution, it seems reasonable, taking into account the known results about the normal law, to search such estimators inside the family of unbiased linear estimators. However, we have proven that it is not possible to obtain efficient linear estimators. Then, we have focused our interest on determining the best unbiased linear estimators in the sense of minimizing the distance to the Cramér-Rao lower bound. The results theoretically obtained are illustrated in a numerical simulation example.
\end{abstract}

Keywords: Estimation; Efficiency property; Kotz type distribution

\section{Introduction}

The elliptically contoured distributions have been in the last years the object of a wide research. This fact is justified for several reasons: on the one hand, this kind of symmetric distributions models a large number of practical situations and, on the other, the family of elliptically contoured distributions includes some of the better known probability distributions (such as the normal law, the uniform one, the stable and logistic laws, Kotz and Bessel distributions and Pearson laws, among others) and it also preserves some of the most important and useful properties of the normal distribution under dependence assumptions between the sample random vectors.

We will specially mention the works presented by Fang and Zhang [1] and Gupta and Varga [2], who collected the most important results on the theory of elliptically contoured

\footnotetext{
*Corresponding author. Tel.: +34-958-243265; Fax: +34-958-243267; Email: rgjaimez@ugr.es
} 
distributions available in the literature and organized them in a unified manner. Another more specific properties of these distributions have been also approached. In this sense, Shapiro and Browne [3] and Sutradhar [4] generalized the analysis of covariance valid for the normal law to the case of elliptical distributions; Golan Kibria and Safiul Haq [5] studied some inference problems in the linear model with errors having an elliptical distribution; Chu [6] and Girón and Rojano [7] approached the estimation problem in stochastic systems assuming elliptically contoured errors, etc.

Our aim in this article is to study the efficiency property of the estimators of the parameters of the bivariate Kotz type distribution inside the family of unbiased linear estimators. For this purpose, we assume that the sample random vectors are jointly distributed as a specific matrix-variate distribution (justified by the form of the bivariate marginal distributions of a Kotz type random matrix). A similar dependence has been recently used by Gutiérrez and Jiménez $[8,9]$ to study the efficiency property in the bivariate Pearson type II and type VII distributions.

Firstly, the Cramér-Rao regularity conditions are analyzed by using the properties of matrix differential calculus [10]. Secondly, from the analogy with the normal distribution, and taking into account the results about efficient estimation in the normal law [11], we construct the family of unbiased linear estimators of the parameters of the bivariate Kotz type distribution.

First of all, it would be reasonable to think that this class of estimators might contain efficient estimators. However, it is proven that this is not true in general. Then, our study is focused on the search of unbiased linear estimators which minimize the distance to the Cramér-Rao lower bound, concluding that the sample mean and the sample covariance matrix, affected by an unbiasedness weighting, satisfy this minimum condition.

\section{Cramér-Rao regularity conditions}

Let us consider $\mathbf{Z}_{1}, \ldots, \mathbf{Z}_{n}$ random vectors identically distributed as a bivariate Kotz type distribution denoted by $K_{2 \times 1}(\boldsymbol{\mu}, \boldsymbol{\Sigma}, q, r, s)$ with $q, r, s \in \mathbb{R}^{+}$, that is, the probability density function of each $\mathbf{Z}_{i}$ is given by

$$
f\left(\mathbf{z}_{\mathbf{i}}\right)=\frac{s r^{(q / s)}}{\pi \Gamma(q / s)|\Sigma|^{1 / 2}}\left[\left(\mathbf{z}_{i}-\boldsymbol{\mu}\right)^{\prime} \boldsymbol{\Sigma}^{-1}\left(\mathbf{z}_{\mathbf{i}}-\boldsymbol{\mu}\right)\right]^{q-1} \exp \left\{-r\left[\left(\mathbf{z}_{\mathbf{i}}-\boldsymbol{\mu}\right)^{\prime} \boldsymbol{\Sigma}^{-1}\left(\mathbf{z}_{\mathbf{i}}-\boldsymbol{\mu}\right)\right]^{s}\right\} .
$$

We are interested in estimating the unknown parameter vector $\theta=\left(\boldsymbol{\mu}^{\prime}, \operatorname{vec}^{\prime}(\boldsymbol{\Sigma})\right.$ where vec $(\cdot)$ denotes the vec operator. As $\boldsymbol{\Sigma}$ is a symmetric matrix, vec $(\boldsymbol{\Sigma})$ contains repeated elements; so, in order to simplify the calculus, we have taken $\mathrm{v}(\boldsymbol{\Sigma})$ (vector formed by the different elements of $\operatorname{vec}(\boldsymbol{\Sigma}))$ and, hence $\theta=\left(\boldsymbol{\mu}^{\prime}, \mathrm{v}^{\prime}(\boldsymbol{\Sigma})\right)$ is the parameter considered to be estimated.

Let $U$ be a random variable independent of $\left(\mathbf{Z}_{1}, \ldots, \mathbf{Z}_{n}\right)$ such that $U^{2}$ is distributed as a beta distribution $\beta(1, n-1)$. Let us assume that $\mathbf{X}=\left(\mathbf{X}_{1}, \ldots, \mathbf{X}_{n}\right)=\left(U \mathbf{Z}_{1}, \ldots, U \mathbf{Z}_{n}\right)$ is distributed as a matrix-variate Kotz type distribution $K_{2 \times n}\left(\boldsymbol{\mu} \mathbf{1}^{\prime}, \boldsymbol{\Sigma} \otimes \mathbf{I}_{n}, q-n+1, r, s\right)$ where $\mathbf{1}^{\prime}=(1, \ldots, 1)$ of dimension $1 \times n, \otimes$ denotes the Kronecker product and $\mathbf{I}_{n}$ the $n \times n$ identity matrix. Then, the joint likelihood function is

$$
\begin{aligned}
L(\mathbf{x} \mid \theta)= & \frac{s r^{(q / s)} \Gamma(n)}{\pi^{n} \Gamma(q / s)|\boldsymbol{\Sigma}|^{n / 2}}\left(\operatorname{tr}\left(\left(\mathbf{x}-\boldsymbol{\mu} \mathbf{1}^{\prime}\right)^{\prime} \boldsymbol{\Sigma}^{-1}\left(\mathbf{x}-\boldsymbol{\mu} \mathbf{1}^{\prime}\right)\right)\right)^{q-n} \\
& \times \exp \left\{-r\left(\operatorname{tr}\left(\left(\mathbf{x}-\boldsymbol{\mu} \mathbf{1}^{\prime}\right)^{\prime} \boldsymbol{\Sigma}^{-1}\left(\mathbf{x}-\boldsymbol{\mu} \mathbf{1}^{\prime}\right)\right)\right)^{s}\right\} .
\end{aligned}
$$

In order to study the Cramér-Rao regularity conditions, next we present some useful results to prove these conditions. 
LEMMA 1 Let us consider $\mathbf{Y}=\left(\mathbf{Y}_{1}, \ldots, \mathbf{Y}_{k}\right) \sim K_{2 \times k}\left(\mathbf{0}, \mathbf{I}_{2} \otimes \mathbf{I}_{k}, q, r, s\right)$ and $\mathbf{A}$ a $2 \times 2$ positive definite matrix. Then, if $\mathbf{X}=\mathbf{A}^{1 / 2} \mathbf{Y}$ (where $\mathbf{A}^{1 / 2}$ denotes the symmetric square root of matrix $\mathbf{A})$, the moment of order $l$ of $\mathbf{X}$, for $l=1,2,3,4$, denoted by

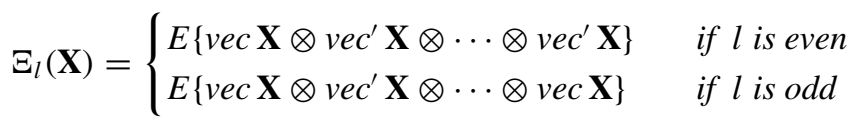

is given as follows

(i)

$$
\Xi_{1}(\mathbf{X})=\mathbf{0}_{2 k \times 1}
$$

(ii)

$$
\Xi_{2}(\mathbf{X})=\frac{\Gamma((q+k) / s) r^{-(1 / s)}}{2 k \Gamma(q+k-1) / s}\left(\mathbf{I}_{k} \otimes \mathbf{A}\right)
$$

(iii)

$$
\Xi_{3}(\mathbf{X})=\mathbf{0}_{4 k^{2} \times 2 k}
$$

(iv)

$$
\begin{aligned}
\Xi_{4}(\mathbf{X})= & \frac{\Gamma(k) \Gamma((q+k+1) / s) r^{-(2 / s)}}{4 \Gamma(k+2) \Gamma(q+k-1) / s}\left(\mathbf{I}_{k} \otimes \mathbf{A}^{1 / 2} \otimes \mathbf{I}_{k} \otimes \mathbf{A}^{1 / 2}\right)\left(2 \mathbf{N}_{2 k}+\operatorname{vec} \mathbf{I}_{2 k} \operatorname{vec}^{\prime} \mathbf{I}_{2 k}\right) \\
& \times\left(\mathbf{I}_{k} \otimes \mathbf{A}^{1 / 2} \otimes \mathbf{I}_{k} \otimes \mathbf{A}^{1 / 2}\right)
\end{aligned}
$$

with $\mathbf{N}_{a}=(1 / 2)\left(\mathbf{I}_{a^{2}}+C_{a}\right)$ being $\mathbf{C}_{a}$ the $a^{2} \times a^{2}$ commutation matrix.

Proof Firstly, from the properties of the Kronecker product [10], the following relations between the moments of $\mathbf{X}$ and those of $\mathbf{Y}$ are deduced

$$
\begin{aligned}
& \Xi_{1}(\mathbf{X})=\left(\mathbf{I}_{k} \otimes \mathbf{A}^{1 / 2}\right) \Xi_{1}(\mathbf{Y}) \\
& \Xi_{2}(\mathbf{X})=\left(\mathbf{I}_{k} \otimes \mathbf{A}^{1 / 2}\right) \Xi_{2}(\mathbf{Y})\left(\mathbf{I}_{k} \otimes \mathbf{A}^{1 / 2}\right) \\
& \Xi_{3}(\mathbf{X})=\left(\mathbf{I}_{k} \otimes \mathbf{A}^{1 / 2} \otimes \mathbf{I}_{k} \otimes \mathbf{A}^{1 / 2}\right) \Xi_{3}(\mathbf{Y})\left(\mathbf{I}_{k} \otimes \mathbf{A}^{1 / 2}\right) \\
& \Xi_{4}(\mathbf{X})=\left(\mathbf{I}_{k} \otimes \mathbf{A}^{1 / 2} \otimes \mathbf{I}_{k} \otimes \mathbf{A}^{1 / 2}\right) \Xi_{4}(\mathbf{Y})\left(\mathbf{I}_{k} \otimes \mathbf{A}^{1 / 2} \otimes \mathbf{I}_{k} \otimes \mathbf{A}^{1 / 2}\right)
\end{aligned}
$$

Secondly, by using the properties of the matrix differential calculus [10], it is obtained that

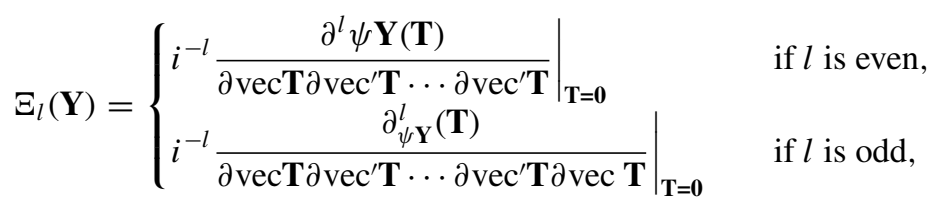

where $\psi_{\mathbf{Y}}(\mathbf{T})$ denotes the characteristic function of $\mathbf{Y}$, which can be expressed as

$$
\psi_{\mathbf{Y}}(\mathbf{T})=\phi\left(\operatorname{tr}\left(\mathbf{T}^{\prime} \mathbf{T}\right)\right)
$$

since it is the characteristic function of a elliptically contoured random matrix [2]. Then, differentiating successively $\psi_{\mathbf{Y}}(\mathbf{T})$ by using the properties of the matrix differential calculus, 
we obtain

$$
\begin{aligned}
& \frac{\partial \psi}{\partial \operatorname{vec} \mathbf{T}}=2 \phi^{\prime} \text { vec } \mathbf{T} \\
& \frac{\partial^{2} \psi}{\partial \operatorname{vec} \mathbf{T} \partial \operatorname{vec}^{\prime} \mathbf{T}}=4 \phi^{\prime \prime} \operatorname{vec}^{\mathbf{T}} \operatorname{vec}^{\prime} \mathbf{T}+2 \phi^{\prime} \mathbf{I}_{2 k} \\
& \frac{\partial^{3} \psi}{\partial \operatorname{vec} \mathbf{T} \partial \operatorname{vec}^{\prime} \mathbf{T} \partial \operatorname{vec} \mathbf{T}}=8 \phi^{\prime \prime \prime}\left(\operatorname{vec} \mathbf{T} \otimes \operatorname{vec}^{\prime} \mathbf{T} \otimes \operatorname{vec} \mathbf{T}\right)+4 \phi^{\prime \prime}\left[\left(\operatorname{vec} \mathbf{T} \otimes \mathbf{I}_{2 k}\right)\right. \\
& \left.+\left(\mathbf{I}_{2 k} \otimes \operatorname{vec} \mathbf{T}\right)\right]+4 \phi^{\prime \prime} \operatorname{vec}_{2 k} \operatorname{vec}^{\prime} \mathbf{T} \\
& \frac{\partial^{4} \psi}{\partial \operatorname{vec} \mathbf{T} \partial \operatorname{vec}^{\prime} \mathbf{T} \partial \operatorname{vec} \mathbf{T} \partial \operatorname{vec}^{\prime} \mathbf{T}}=16 \phi^{i v}\left(\operatorname{vec} \mathbf{T} v^{\prime} c^{\prime} \mathbf{T} \otimes \operatorname{vec} \mathbf{T} v e c^{\prime} \mathbf{T}\right)+8 \phi^{\prime \prime \prime}\left[\left(\operatorname{vec} \mathbf{T} \operatorname{vec}^{\prime} \mathbf{T} \otimes \mathbf{I}_{2 k}\right)\right. \\
& \left.+\left(\operatorname{vec}^{\prime} \mathbf{T} \otimes \operatorname{vec} \mathbf{I}_{2 k} \otimes \operatorname{vec}^{\prime} \mathbf{T}\right)+\mathbf{I}_{2 k} \otimes \operatorname{vec}^{\prime} \mathbf{v} \operatorname{vec}^{\prime} \mathbf{T}\right] \\
& +8 \phi^{\prime \prime \prime}\left(\operatorname{vec} \mathbf{T} \otimes \operatorname{vec}^{\prime} \mathbf{I}_{2 k} \otimes \operatorname{vec} \mathbf{T}\right)+4 \phi^{\prime \prime} \mathbf{C}_{2 k}+4 \phi^{\prime \prime} \mathbf{I}_{4 k^{2}} \\
& +8 \phi^{\prime \prime \prime}\left(\mathbf{I}_{2 k} \otimes \operatorname{vec}^{\mathbf{T} v e c}{ }^{\prime} \mathbf{T}+4 \phi^{\prime \prime} \operatorname{vec}_{2 k} \operatorname{vec}^{\prime} \mathbf{I}_{2 k}\right) \\
& +4 \phi^{\prime \prime \prime}\left[\left(\operatorname{vec}^{\prime} \mathbf{T} \otimes \mathbf{I}_{2 k} \otimes \operatorname{vec} \mathbf{T}\right)+\left(\operatorname{vec} \mathbf{T} \otimes \mathbf{I}_{2 k} \otimes \operatorname{vec}^{\prime} \mathbf{T}\right)\right] .
\end{aligned}
$$

By using equations (6)-(10), the moments $\Xi_{l}(\mathbf{Y}), l=1, \ldots, 4$ are obtained. Finally, from them and equations (2)- (5), the required results are derived, taking into account that $-2 \phi^{\prime}(0)=$ $E\left[Y_{11}^{2}\right]$ and $4 \phi^{\prime \prime}(0)=\frac{1}{3} E\left[Y_{11}^{4}\right]$ being $Y_{11}$ the $(1,1)$-element of $\mathbf{Y}$.

LEMMA 2 Let us consider $\mathbf{X}=\left(\mathbf{X}_{1}, \ldots, \mathbf{X}_{n}\right) \sim K_{2 \times n}\left(\boldsymbol{\mu} \mathbf{1}^{\prime}, \mathbf{\Sigma} \otimes \mathbf{I}_{n}, q, r,\right)$ such that $q+n>$ 1 and $r, s \in \mathbb{R}^{+}$. Let $h: \mathbb{R}^{2 \times p} \rightarrow \mathbb{R}^{u \times v}$ be a function with $p, u, v \in \mathbb{N}$, and $i_{1}, \ldots, i_{p} \in$ $\{1, \ldots, n\}, p$ different values. Then

(i) $E_{\mathbf{X}}\left\{h\left(\boldsymbol{\Sigma}^{-1}\left(\mathbf{X}_{i_{1}}-\boldsymbol{\mu}\right), \ldots, \boldsymbol{\Sigma}^{-1}\left(\mathbf{X}_{i_{p}}-\boldsymbol{\mu}\right)\right)\right\}=E_{\mathbf{Y}}\left\{h\left(\boldsymbol{\Sigma}^{-1 / 2} \mathbf{Y}_{i_{1}}, \ldots, \boldsymbol{\Sigma}^{-1 / 2} \mathbf{Y}_{i_{p}}\right)\right\}$

(ii) $E_{\mathbf{X}}\left\{h\left(\left(\mathbf{X}_{i_{1}}-\boldsymbol{\mu}\right), \ldots,\left(\mathbf{X}_{i_{p}}-\boldsymbol{\mu}\right)\right)\right\}=E_{\mathbf{Y}}\left\{h\left(\boldsymbol{\Sigma}^{1 / 2} \mathbf{Y}_{i_{1}}, \ldots, \boldsymbol{\Sigma}^{1 / 2} \mathbf{Y}_{i_{p}}\right)\right\}$

where $\mathbf{Y}=\left(\mathbf{Y}_{i_{1}}, \ldots, \mathbf{Y}_{i_{p}}\right)=U \mathbf{Z}$, being $U$ and $\mathbf{Z}$ independent with $U^{2} \sim \beta(p, n-p)$ and $\mathbf{Z}=\left(\mathbf{Z}_{i_{1}}, \ldots, \mathbf{Z}_{i_{p}}\right) \sim K_{2 \times p}\left(\mathbf{0}, \mathbf{I}_{2} \otimes \mathbf{I}_{p}, q+n-p, r, s\right)$.

Proof We show the proof of the first property as the second one is obtained in an analogous way. From equation (1), it is clear that

$$
\begin{aligned}
E_{\mathbf{X}}\left\{h\left(\boldsymbol{\Sigma}^{-1}\left(\mathbf{X}_{i_{1}}-\boldsymbol{\mu}\right), \ldots, \boldsymbol{\Sigma}^{-1}\left(\mathbf{X}_{i_{p}}-\boldsymbol{\mu}\right)\right)\right\} \\
=c_{0} \int_{\mathbb{R}^{2 \times n}} h\left(\boldsymbol{\Sigma}^{-1}\left(\mathbf{x}_{i_{1}}-\boldsymbol{\mu}\right), \ldots, \boldsymbol{\Sigma}^{-1}\left(\mathbf{x}_{i_{p}}-\boldsymbol{\mu}\right)\right)\left(\sum_{i=1}^{n}\left(\mathbf{x}_{i}-\boldsymbol{\mu}\right)^{\prime} \boldsymbol{\Sigma}^{-1}\left(\mathbf{x}_{i}-\boldsymbol{\mu}\right)\right)^{q-1} \\
\quad \times \exp \left\{-r\left(\sum_{i=1}^{n}\left(\mathbf{x}_{i}-\boldsymbol{\mu}\right)^{\prime} \boldsymbol{\Sigma}^{-1}\left(\mathbf{x}_{i}-\boldsymbol{\mu}\right)\right)\right\} \bigwedge_{i=1}^{n} \mathrm{~d} \mathbf{x}_{i}
\end{aligned}
$$

begin $c_{0}=\frac{s r^{(q+n-1) / s} \Gamma(n)}{\pi^{n} \Gamma(q+n-1) / s|\Sigma|^{n / 2}}$. 
Let $I$ be the integral appearing on the right-hand side of the earlier expression, that is,

$$
\begin{aligned}
I= & \int_{\mathbb{R}^{2 \times n}} h\left(\boldsymbol{\Sigma}^{-1}\left(\mathbf{x}_{i_{1}}-\boldsymbol{\mu}\right), \ldots, \boldsymbol{\Sigma}^{-1}\left(\mathbf{x}_{i_{p}}-\boldsymbol{\mu}\right)\right)\left(\sum_{i=1}^{n}\left(\mathbf{x}_{i}-\boldsymbol{\mu}\right)^{\prime} \boldsymbol{\Sigma}^{-1}\left(\mathbf{x}_{i}-\boldsymbol{\mu}\right)\right)^{q-1} \\
& \times \exp \left\{-r\left(\sum_{i=1}^{n}\left(\mathbf{x}_{i}-\boldsymbol{\mu}\right)^{\prime} \boldsymbol{\Sigma}^{-1}\left(\mathbf{x}_{i}-\boldsymbol{\mu}\right)\right)^{s}\right\} \bigwedge_{i=1}^{n} \mathrm{~d} \mathbf{x}_{i}
\end{aligned}
$$

For each $k \in\{1, \ldots, n\}$ with $k \neq i_{j}, j=l, \ldots, p$, we consider the transformation

$$
\mathbf{z}_{k}=\left(\sum_{j=1}^{p}\left(\mathbf{x}_{i_{j}}-\boldsymbol{\mu}\right)^{\prime} \boldsymbol{\Sigma}^{-1}\left(\mathbf{x}_{i_{j}}-\boldsymbol{\mu}\right)\right)^{1 / 2} \boldsymbol{\Sigma}^{-1 / 2}\left(\mathbf{x}_{k}-\boldsymbol{\mu}\right) .
$$

Then, $I$ can be rewritten as

$$
\begin{aligned}
& c_{1} \int_{\mathbb{R}^{2 \times p}} \int_{\mathbb{R}^{2 \times(n-p)}} h\left(\boldsymbol{\Sigma}^{-1}\left(\mathbf{x}_{i_{1}}-\boldsymbol{\mu}\right), \ldots, \boldsymbol{\Sigma}^{-1}\left(\mathbf{x}_{i_{p}}-\boldsymbol{\mu}\right)\right) \\
& \quad \times\left(\sum_{j=1}^{p}\left(\mathbf{x}_{i_{j}}-\boldsymbol{\mu}\right)^{\prime} \boldsymbol{\Sigma}^{-1}\left(\mathbf{x}_{i_{j}}-\boldsymbol{\mu}\right)\right)^{q+n-p-1} \\
& \quad \times \exp \left\{-r\left(\sum_{j=1}^{p}\left(\mathbf{x}_{i_{j}}-\boldsymbol{\mu}\right)^{\prime} \boldsymbol{\Sigma}^{-1}\left(\mathbf{x}_{i_{j}}-\boldsymbol{\mu}\right)\right)^{s}\left(1+\sum_{\substack{k=1 \\
k \neq i_{1}, \ldots, i_{p}}}^{n} \mathbf{z}_{k}^{\prime} \mathbf{z}_{k}\right)^{s}\right\} \\
& \quad \times\left(1+\sum_{\substack{k=1 \\
k \neq i_{1}, \ldots, i_{p}}}^{n} \mathbf{z}_{k}^{\prime} \mathbf{z}_{k} \bigwedge_{\substack{k=1 \\
k \neq i_{1}, \ldots, i_{p}}}^{n} \mathrm{~d} \mathbf{z}_{k} \bigwedge_{j=1}^{p} \mathrm{~d} \mathbf{x}_{i_{j}}\right.
\end{aligned}
$$

where $c_{1}=|\boldsymbol{\Sigma}|^{(n-p) / 2}$.

Next, denoting by $I_{1}$ the integral below

$$
\begin{aligned}
I_{1}= & \int_{\mathbb{R}^{2 \times(n-p)}} \exp \left\{-r\left(\sum_{j=1}^{p}\left(\mathbf{x}_{i_{j}}-\boldsymbol{\mu}\right)^{\prime} \boldsymbol{\Sigma}^{-1}\left(\mathbf{x}_{i_{j}}-\boldsymbol{\mu}\right)\right)^{s}\left(1+\sum_{\substack{k=1 \\
k \neq i_{1}, \ldots, i_{p}}}^{n} \mathbf{z}_{k}^{\prime} \mathbf{z}_{k}\right)^{s}\right\} \\
& \times\left(1+\sum_{\substack{k=1 \\
k \neq i_{1}, \ldots, i_{p}}}^{n} \mathbf{z}_{k}^{\prime} \mathbf{z}_{k}\right)^{q-1} \bigwedge_{\substack{k=1 \\
k \neq i_{1}, \ldots, i_{p}}}^{n} \mathrm{~d} \mathbf{z}_{k}
\end{aligned}
$$

and considering, for each $k \in\{1, \ldots, n\}$ with $k \neq i_{j}, j=1, \ldots, p$ the transformation given by $\mathbf{z}_{k}=\left(t_{k} \cos \theta_{k}, t_{k} \sin \theta_{k}\right)^{\prime}$ where $t_{k} \in \mathbb{R}^{+}, \theta_{k} \in(0,2 \pi]$, it is deduced that $I_{1}$ is equivalent to 
this integral

$$
\begin{aligned}
& (2 \pi)^{n-p} \int_{\left(\mathbb{R}^{+}\right)^{n-p}} \exp \left\{-r\left(\sum_{j=1}^{p}\left(\mathbf{x}_{i_{j}}-\boldsymbol{\mu}\right)^{\prime} \boldsymbol{\Sigma}^{-1}\left(\mathbf{x}_{i_{j}}-\boldsymbol{\mu}\right)\right)^{s}\left(1+\sum_{\substack{k=1 \\
k \neq i_{1}, \ldots, i_{p}}}^{n} t_{k}^{2}\right\}\right. \\
& \quad \times\left(1+\sum_{\substack{k=1 \\
k \neq i_{1}, \ldots, i_{p}}}^{n} t_{k}^{2}\right)^{q-1} \prod_{\substack{k=1 \\
k \neq i_{1}, \ldots, i_{p}}}^{n} t_{k} \bigwedge_{\substack{k=1 \\
k \neq i_{1}, \ldots, i_{p}}}^{n} \mathrm{~d} t_{k} .
\end{aligned}
$$

Using the transformation to polar coordinates

$$
\begin{array}{rl|r}
t_{j_{1}} & =t \cos \theta_{1} & \\
t_{j_{k}} & =t \prod_{i=1}^{k-1} \sin \theta_{i} \cos \theta_{k} \quad(2 \leq k \leq n-p-1) & \begin{array}{r}
t \in \mathbb{R}^{+}, \theta_{i} \in\left(0, \frac{\pi}{2}\right] \\
i=1, \ldots, n-p-1,
\end{array} \\
t_{j_{n-p}}=t \prod_{i=1}^{n-p-1} \sin \theta_{i} &
\end{array}
$$

it is obtained that equation (12) can be rewritten as follows

$$
\begin{aligned}
& (2 \pi)^{n-p} \int_{\mathbb{R}^{+}}\left(1+t^{2}\right)^{q-1} t^{2 n-2 p-1} \exp \left\{-r\left(\sum_{j=1}^{p}\left(\mathbf{x}_{i_{j}}-\boldsymbol{\mu}\right)^{\prime} \boldsymbol{\Sigma}^{-1}\left(\mathbf{x}_{i_{j}}-\boldsymbol{\mu}\right)\right)^{s}\left(1+t^{2}\right)^{s}\right\} \mathrm{d} t \\
& \quad \times \prod_{i=1}^{n-p-1} \int_{0}^{\pi / 2} \sin ^{2(n-p-i)-1} \theta_{i} \cos \theta_{i} \mathrm{~d} \theta_{i}
\end{aligned}
$$

Consequently,

$$
\begin{aligned}
I_{1}= & \frac{2 \pi^{n-p}}{\Gamma(n-p)} \int_{\mathbb{R}^{+}}\left(1+t^{2}\right)^{q-1} t^{2 n-2 p-1} \\
& \times \exp \left\{-r\left(\sum_{j=1}^{p}\left(\mathbf{x}_{i_{j}}-\boldsymbol{\mu}\right)^{\prime} \boldsymbol{\Sigma}^{-1}\left(\mathbf{x}_{i_{j}}-\boldsymbol{\mu}\right)\right)^{s}\left(1+t^{2}\right)^{s}\right\} \mathrm{d} t
\end{aligned}
$$

and hence, equation (11) can be expressed as

$$
\begin{array}{rl}
c_{2} \int_{\mathbb{R}^{2 \times p}} & h\left(\boldsymbol{\Sigma}^{-1}\left(\mathbf{x}_{i_{1}}-\boldsymbol{\mu}\right), \ldots, \boldsymbol{\Sigma}^{-1}\left(\mathbf{x}_{i_{p}}-\boldsymbol{\mu}\right)\right) \\
\quad \times\left(\sum_{j=1}^{p}\left(\mathbf{x}_{i_{j}}-\boldsymbol{\mu}\right)^{\prime} \boldsymbol{\Sigma}^{-1}\left(\mathbf{x}_{i_{j}}-\boldsymbol{\mu}\right)\right)^{q+n-p-1} \int_{\mathbb{R}^{+}}\left(1+t^{2}\right)^{q-1} t^{2 n-2 p-1} \\
\quad \times \exp \left\{-r\left(\sum_{j=1}^{p}\left(\mathbf{x}_{i_{j}}-\boldsymbol{\mu}\right)^{\prime} \boldsymbol{\Sigma}^{-1}\left(\mathbf{x}_{i_{j}}-\boldsymbol{\mu}\right)\right)^{s}\left(1+t^{2}\right)^{s}\right\} \mathrm{d} t \bigwedge_{j=1}^{p} \mathrm{~d} \mathbf{x}_{i_{j}},
\end{array}
$$


where

$$
c_{2}=\frac{2 \pi^{n-p}|\boldsymbol{\Sigma}|^{(n-p) / 2}}{\Gamma(n-p)} .
$$

Next, for each $i_{j}, j=l, \ldots, p$, we consider the transformation $y_{i_{j}}=\boldsymbol{\Sigma}^{-1 / 2}\left(\mathbf{x}_{i_{j}}-\boldsymbol{\mu}\right)$; so equation (13) can be rewritten as follows

$$
\begin{aligned}
& c_{3} \int_{\mathbb{R}^{2 \times p}} h\left(\boldsymbol{\Sigma}^{-1 / 2} \mathbf{y}_{i_{1}}, \ldots, \boldsymbol{\Sigma}^{-1 / 2} \mathbf{y}_{i_{p}}\right)\left(\sum_{j=1}^{p} \mathbf{y}_{i_{j}}^{\prime} \mathbf{y}_{i_{j}}\right)^{q+n-p-1} \\
& \quad \times \int_{\mathbb{R}^{+}}\left(1+t^{2}\right)^{q-1} t^{2 n-2 p-1} \exp \left\{-r\left(\sum_{j=1}^{p} \mathbf{y}_{i_{j}}^{\prime} \mathbf{y}_{i_{j}}\right)^{s}\left(1+t^{2}\right)^{s}\right\} \mathrm{d} t \bigwedge_{j=1}^{p} \mathrm{~d} \mathbf{y}_{i_{j}},
\end{aligned}
$$

where

$$
c_{3}=\frac{2 \pi^{n-p}|\Sigma|^{n / 2}}{\Gamma(n-p)} .
$$

Finally, the desired result is deduced taking into account that if $\mathbf{Y}=\left(\mathbf{Y}_{i_{j}}\right)_{j=1, \ldots, p}=U \mathbf{Z}$, being $U$ and $\mathbf{Z}$ independent with $\mathbf{Z}=\left(\mathbf{Z}_{i_{j}}\right)_{j=1, \ldots, p} \sim K_{2 \times p}\left(\mathbf{0}, \mathbf{I}_{2} \otimes \mathbf{I}_{n-p}, q+n-p, r, s\right)$ and $U^{2} \sim \beta(p, n-p)$, the integral $I$ can be expressed as

$$
\begin{aligned}
I & =\frac{\pi^{n} \Gamma((q+n-1) / s)|\boldsymbol{\Sigma}|^{n / 2}}{s r^{(q+n-1) / s} \Gamma(n)} \int_{\mathbb{R}^{2 \times p}} h\left(\boldsymbol{\Sigma}^{-1 / 2} \mathbf{y}_{i_{1}}, \ldots, \boldsymbol{\Sigma}^{-1 / 2} \mathbf{y}_{i_{p}}\right) f\left(\mathbf{y}_{i_{1}}, \ldots, \mathbf{y}_{i_{p}}\right) \bigwedge_{j=1}^{p} \mathrm{~d} \mathbf{y}_{i_{j}} \\
& =\frac{1}{c_{0}} E_{\mathbf{Y}}\left\{h\left(\boldsymbol{\Sigma}^{-1 / 2} \mathbf{Y}_{i_{1}}, \ldots, \boldsymbol{\Sigma}^{-1 / 2} \mathbf{Y}_{i_{p}}\right)\right\}
\end{aligned}
$$

THEOREM 1 Under the initial conditions, let us define $\mathbf{Y}_{i}=\boldsymbol{\Sigma}^{-1 / 2}\left(\mathbf{X}_{i}-\boldsymbol{\mu}\right)$. Then, for each $i \in\{1, \ldots, n\}$ and $m \in \mathbb{N}$ such that $m<q$, we have

(i)

$$
E_{\mathbf{X}}\left\{\left(\sum_{k=1}^{n} \mathbf{Y}_{k}^{\prime} \mathbf{Y}_{k}\right)^{-m} \boldsymbol{\Sigma}^{-1 / 2} \mathbf{Y}_{i}\right\}=\mathbf{0}_{2 \times 1}
$$

(ii)

$$
E_{\mathbf{X}}\left\{\left(\sum_{k=1}^{n} \mathbf{Y}_{k}^{\prime} \mathbf{Y}_{k}\right)^{-m} \boldsymbol{\Sigma}^{-1 / 2} \mathbf{Y}_{i} \mathbf{Y}_{i}^{\prime} \boldsymbol{\Sigma}^{-1 / 2}\right\}=\frac{\Gamma((q-m+1) / s) r^{(m-1) / s}}{2 n \Gamma(q / s)} \boldsymbol{\Sigma}^{-1}
$$

(iii)

$$
E_{\mathbf{X}}\left\{\left(\sum_{k=1}^{n} \mathbf{Y}_{k}^{\prime} \mathbf{Y}_{k}\right)^{-m} \boldsymbol{\Sigma}^{-1 / 2} \mathbf{Y}_{i} \operatorname{vec}^{\prime}\left(\boldsymbol{\Sigma}^{-1 / 2} \mathbf{Y}_{i} \mathbf{Y}_{i}^{\prime} \boldsymbol{\Sigma}^{-1 / 2}\right)\right\}=\mathbf{0}_{2 \times 4}
$$

(iv)

$$
\begin{aligned}
E_{\mathbf{X}} & \left\{\left(\sum_{k=1}^{n} \mathbf{Y}_{k}^{\prime} \mathbf{Y}_{k}\right)^{-m} \operatorname{vec}\left(\boldsymbol{\Sigma}^{-1 / 2} \mathbf{Y}_{i} \mathbf{Y}_{i}^{\prime} \boldsymbol{\Sigma}^{-1 / 2}\right) \operatorname{vec}^{\prime}\left(\boldsymbol{\Sigma}^{-1 / 2} \mathbf{Y}_{i} \mathbf{Y}_{i}^{\prime} \boldsymbol{\Sigma}^{-1 / 2}\right)\right\} \\
& =\frac{\Gamma((q-m+2) / s) r^{(m-2) / s}}{4 n(n+1) \Gamma(q / s)}\left(\boldsymbol{\Sigma}^{-1 / 2} \otimes \boldsymbol{\Sigma}^{-1 / 2}\right)\left(2 \mathbf{N}_{2}+\operatorname{vec}_{2} \operatorname{vec}^{\prime} \mathbf{I}_{2}\right)\left(\boldsymbol{\Sigma}^{-1 / 2} \otimes \boldsymbol{\Sigma}^{-1 / 2}\right) .
\end{aligned}
$$


Proof Let us consider a function $h: \mathbb{R}^{2} \rightarrow \mathbb{R}^{u \times v}$. Then, from Lemma 2, we have that

$$
\begin{aligned}
E_{\mathbf{X}} & \left\{\left(\sum_{k=1}^{n}\left(\mathbf{X}_{k}-\boldsymbol{\mu}\right)^{\prime} \boldsymbol{\Sigma}^{-1}\left(\mathbf{X}_{k}-\boldsymbol{\mu}\right)\right)^{-m} h\left(\boldsymbol{\Sigma}^{-1}\left(\mathbf{X}_{i}-\boldsymbol{\mu}\right)\right)\right\} \\
& =\frac{\Gamma((q-m) / s) r^{m / s}}{\Gamma(q / s)} E_{\mathbf{Y}_{i}}\left\{h\left(\boldsymbol{\Sigma}^{-1 / 2} \mathbf{Y}_{i}\right)\right\}
\end{aligned}
$$

being $\mathbf{Y}_{i}=U \mathbf{Z}_{i}$ with $U$ and $\mathbf{Z}_{i}$ independent and such that $\mathbf{Z}_{i} \sim K_{2 \times 1}\left(\mathbf{0}, \mathbf{I}_{2}, q-m, r, s\right)$ and $U^{2} \sim \beta(1, n-1)$. Then, the result is easily obtained as a consequence of Lemma 1 .

THEOREM 2 Under the initial conditions, let us define $\mathbf{Y}_{i}=\boldsymbol{\Sigma}^{-1 / 2}\left(\mathbf{X}_{i}-\boldsymbol{\mu}\right)$. Then, if $i, j \in$ $\{1, \ldots, n\}$ with $i \neq j$ and $m \in \mathbb{N}$, such that $m<q$. we have

(ii)

$$
E_{\mathbf{X}}\left\{\left(\sum_{k=1}^{n} \mathbf{Y}_{k}^{\prime} \mathbf{Y}_{k}\right)^{-m} \boldsymbol{\Sigma}^{-1 / 2} \mathbf{Y}_{j}^{\prime} \boldsymbol{\Sigma}^{-1 / 2}\right\}=0_{2 \times 2}
$$

$$
E_{\mathbf{X}}\left\{\left(\sum_{k=1}^{n} \mathbf{Y}_{k}^{\prime} \mathbf{Y}_{k}\right)^{-m} \boldsymbol{\Sigma}^{-1 / 2} \mathbf{Y}_{i} \operatorname{vec}^{\prime}\left(\boldsymbol{\Sigma}^{-1 / 2} \mathbf{Y}_{j} \mathbf{Y}_{j}^{\prime} \boldsymbol{\Sigma}^{-1 / 2}\right)\right\}=0_{2 \times 4}
$$

(iii)

$$
\begin{aligned}
& E_{\mathbf{X}}\left\{\left(\sum_{k=1}^{n} \mathbf{Y}_{k}^{\prime} \mathbf{Y}_{k}\right)^{-m} \operatorname{vec}^{\prime}\left(\boldsymbol{\Sigma}^{-1 / 2} \mathbf{Y}_{i} \mathbf{Y}_{i}^{\prime} \boldsymbol{\Sigma}^{-1 / 2}\right) \operatorname{vec}^{\prime}\left(\boldsymbol{\Sigma}^{-1 / 2} \mathbf{Y}_{j} \mathbf{Y}_{j}^{\prime} \boldsymbol{\Sigma}^{-1 / 2}\right)\right\} \\
& =\frac{\Gamma((q-m+2) / s) r(m-2) / s}{4 n(n+1) \Gamma(q / s)} \operatorname{vec} \boldsymbol{\Sigma}^{-1} \operatorname{vec}^{\prime} \boldsymbol{\Sigma}^{-1} .
\end{aligned}
$$

Proof By using a similar reasoning to that used in the proof of the previous theorem, given a function $h: \mathbb{R}^{2 \times 2} \rightarrow \mathbb{R}^{u \times v}$, from Lemma 2 we have

$E_{\mathbf{X}}\left\{\frac{h\left(\boldsymbol{\Sigma}^{-1}\left(\mathbf{X}_{i}-\boldsymbol{\mu}\right), \boldsymbol{\Sigma}^{-1}\left(\mathbf{X}_{j}-\boldsymbol{\mu}\right)\right)}{\left(\sum_{k=1}^{n}\left(\mathbf{X}_{k}-\boldsymbol{\mu}\right)^{\prime} \boldsymbol{\Sigma}^{-1}\left(\mathbf{X}_{k}-\boldsymbol{\mu}\right)\right)^{m}}\right\}=\frac{\Gamma((q-m) / s) r(m / s)}{\Gamma\lceil(q / s)} E_{\mathbf{Y}}\left\{h\left(\boldsymbol{\Sigma}^{-1 / 2} \mathbf{Y}_{i}, \boldsymbol{\Sigma}^{-1 / 2} \mathbf{Y}_{j}\right)\right\}$

being $\mathbf{Y}=\left(\mathbf{Y}_{i}, \mathbf{Y}_{j}\right)=U \mathbf{Z}$ with $U$ and $\mathbf{Z}$ independent and such that $U^{2} \sim \beta(2, n-2)$ and $\mathbf{Z}=\left(\mathbf{Z}_{i}, \mathbf{Z}_{j}\right) \sim K_{2 \times 2}\left(\mathbf{0}, \mathbf{I}_{2} \otimes \mathbf{I}_{2}, q-m-1, r, s\right)$. Hence, as a consequence of Lemma 1 the established properties are easily derived.

The earlier results guarantee that the Cramér-Rao regularity conditions are satisfied, being the Fisher information matrix

$$
I_{n}^{K}\left(\boldsymbol{\mu}^{\prime}, \mathrm{v}^{\prime}(\boldsymbol{\Sigma})\right)=\left(\begin{array}{c|c}
I_{n}^{K}\left(\boldsymbol{\mu}^{\prime}\right) & \mathbf{0}_{2 \times 3} \\
\hline \mathbf{0}_{3 \times 2} & I_{n}^{K}\left(\mathrm{v}^{\prime}(\boldsymbol{\Sigma})\right)
\end{array}\right)
$$

where

$$
\begin{aligned}
I_{n}^{K}(\boldsymbol{\mu})^{\prime} & =2 r^{(1 / s)} \frac{\Gamma(q-1) / s}{\Gamma(q / s)}\left[(n-1)^{2}+s(q-1)\right] \boldsymbol{\Sigma}^{-1} \\
I_{n}^{K}\left(\mathrm{v}^{\prime}(\boldsymbol{\Sigma})\right) & =\frac{1}{4} \mathbf{Q}\left[\left(-n^{2}+n \frac{n^{2}+q s}{n+1}\right) \operatorname{vec} \boldsymbol{\Sigma}^{-1} \operatorname{vec}^{\prime} \boldsymbol{\Sigma}^{-1}+\frac{n^{2}+q s}{n+1}\left(\boldsymbol{\Sigma}^{-1} \otimes \boldsymbol{\Sigma}^{-1}\right) 2 \mathrm{~N}_{2}\right] \mathbf{Q}^{\prime}
\end{aligned}
$$

with $\mathbf{Q}=\left(\mathbf{E}_{11}+\mathbf{E}_{22}+\mathbf{E}_{34}\right) \in \mathbb{R}^{3 \times 4}\left(\mathbf{E}_{i j}\right.$ denotes the elemental matrix of dimension $3 \times 4$ whose $i j$ th element is the unit and the remaining elements are all zero). 
In view, if the Fisher information matrix obtained, we may remark that it presents a similar structure to that obtained in the normal case assuming independence between the random vectors. More specifically, the submatrices situated in the diagonal, $I_{n}^{K}\left(\boldsymbol{\mu}^{\prime}\right)$ and $I_{n}^{K}\left(\mathrm{v}^{\prime}(\boldsymbol{\Sigma})\right)$, correspond to the Fisher information matrices associated with the estimators of the individual parameters $\mu$ and $\mathrm{v}(\boldsymbol{\Sigma})$, respectively, and the remaining ones are zero matrices. The structure of this matrix is very useful to study the efficiency property of the estimators of the joint parameter $\left(\boldsymbol{\mu}^{\prime}, \mathrm{v}^{\prime}(\boldsymbol{\Sigma})\right)^{\prime}$.

\section{Unbiased linear estimators of minimum distance}

\subsection{Estimators of $\mu$}

As we have previously mentioned, the normal law is a particular Kotz type distribution. For this reason, it is reasonable to think that the sample mean, $\overline{\mathbf{X}}$, may be an efficient estimator of $\boldsymbol{\mu}$. However, we have proven that this estimator does not satisfy the efficiency property. Actually, the determinant of the sample mean covariance matrix, for $q>1$, is given by

$$
\operatorname{det}(\operatorname{Cov}(\overline{\mathbf{X}}, \boldsymbol{\mu}))=\frac{\Gamma((q+1) / s)^{2} r^{-(2 / s)}}{4 n^{4} \Gamma(q / s)^{2}} \operatorname{det} \boldsymbol{\Sigma}
$$

Then, if $q>2$, the associated efficiency equation is

$$
\frac{\Gamma(q / s)^{4}}{\Gamma((q-1) / s)^{2} \Gamma((q+1) / s)^{2}}=\frac{\left((n-1)^{2}+s(q-1)\right)^{2}}{n^{4}}
$$

The following result guarantees that there are not solutions of this equation.

THEOREM 3 For all $n \in \mathbb{N}$ and $q, s \in \mathbb{R}^{+}$, with $q>2$, the following inequality holds

$$
\frac{\Gamma(q / s)^{2}}{\Gamma((q-1) / s) \Gamma(q+1) / s}<\frac{(n-1)^{2}+s(q-1)}{n^{2}}
$$

Proof Firstly, from the definition of the Gamma function due to Weierstrass we obtain

$$
\frac{\Gamma(q / s)^{2}}{\Gamma((q-1) / s) \Gamma(q+1) / s}=\frac{q^{2}-1}{q^{2}} \prod_{n=1}^{\infty}\left(1-\frac{1}{(q+s n)^{2}}\right)
$$

Secondly, if $k \in \mathbb{N}$, the product in the second term of equation (15) can be bounded as follows

$$
\begin{aligned}
\prod_{n=1}^{\infty}\left(1-\frac{1}{(q+s n)^{2}}\right) & <\left(1-\frac{1}{(q+s)^{2}}\right)^{k} \prod_{n=1}^{\infty}\left(1-\frac{1}{(q+s)^{2} n^{2}}\right) \\
& =\left(1-\frac{1}{(q+s)^{2}}\right)^{k} \frac{\sin \pi /(q+s)}{\pi(q+s)}
\end{aligned}
$$

Finally, we have proven that for each $q>2$ and $s \in \mathbb{R}^{+}$there exists $k \in \mathbb{N}$ satisfying

$$
\frac{q^{2}-1}{q^{2}}\left(1-\frac{1}{(q+s)^{2}}\right)^{k} \frac{\sin \pi /(q+s)}{\pi /(q+s)} \leq \frac{(n-1)^{2}+s(q-1)}{n^{2}}, \quad \forall n \in \mathbb{N}
$$


As a consequence of this result, we have tried to search efficient estimators inside the family of unbiased linear estimators of $\mu$, defined by

$$
\mathcal{F}_{\mu}^{K}=\left\{\hat{\boldsymbol{\mu}}=\left(\sum_{i=1}^{n} \alpha_{i}\right)^{-1} \sum_{i=1}^{n} \alpha_{i} \mathbf{X}_{i} / \alpha_{1}, \ldots, \alpha_{n} \in \mathbb{R}\right\}
$$

but we have proven that it is not possible to find such estimators. In fact, for each $\hat{\mu} \in \mathcal{F}_{\mu}^{K}$, the efficiency equation associated, for $q>2$, is

$$
\frac{\Gamma(q / s)^{4}}{\Gamma((q-1) / s)^{2} \Gamma\left((q+1)^{2} / s\right)^{2}} \frac{n^{2}}{\left((n-1)^{2}+s(q-1)\right)^{2}}=\left(\sum_{i=1}^{n} \alpha_{i}\right)^{-4}\left(\sum_{i=1}^{n} \alpha_{i}^{2}\right)^{2}
$$

and, by using Theorem 3, taking into account the following property

$$
\min _{\alpha_{1}, \ldots, \alpha_{n}}\left(\sum_{i=1}^{n} \alpha_{i}\right)^{-2} \sum_{i=1}^{n} \alpha_{i}^{2}=\frac{1}{n}
$$

we can guarantee that there are not efficient linear estimators of $\boldsymbol{\mu}$.

Then, we have focused our interest on determining the best unbiased linear estimators of $\mu$ in the sense of minimizing the distance to the Cramér-Rao lower bound. So, we have proven that, for $q>2$,

$$
\min \left\{\frac{\operatorname{det}(\operatorname{Cov}(\hat{\boldsymbol{\mu}}, \boldsymbol{\mu}))}{\hat{\boldsymbol{\mu}} \in \mathcal{F}_{\boldsymbol{\mu}}^{K}}\right\}=\frac{\Gamma((q+1) / s)^{2} r^{-(2 / s)}}{4 n^{4} \Gamma(q / s)^{2}} \operatorname{det}(\boldsymbol{\Sigma})
$$

Hence, from equation (14), we can conclude that the sample mean is an unbiased linear estimator which minimizes the distance to the Cramér-Rao lower bound.

\subsection{Estimators of $\Sigma$}

Taking again into account the analogy with the normal law, it would be reasonable to think that the sample covariance matrix, affected by an unbiasedness weighting, defined by

$$
\hat{\mathbf{\Sigma}}^{*}=\frac{2 n \Gamma(q / s)}{(n-1) \Gamma((q+1) / s)} \sum_{i=1}^{n}\left(\mathbf{X}_{i}-\overline{\mathbf{X}}\right)\left(\mathbf{X}_{i}-\overline{\mathbf{X}}\right)^{\prime}
$$

could be an efficient estimator. However, we have proven that this estimator is not efficient in general.

In order to prove this property, next we show a result which will be useful to obtain the covariance matrices of the estimators.

THEOREM 4 Under the initial conditions, let us define $\mathbf{Y}_{i}=\mathbf{X}_{i}-\boldsymbol{\mu}$. Then, for $q>1$ and $i_{1}, i_{2}, i_{3}, i_{4} \in\{1, \ldots, n\}$ we have

(i)

$$
E_{\mathbf{X}}\left\{\operatorname{vec}\left(\mathbf{Y}_{i_{j}} \mathbf{Y}_{i_{k}}^{\prime}\right) \operatorname{vec}^{\prime}\left(\mathbf{Y}_{i_{j}} \mathbf{Y}_{i_{k}}^{\prime}\right)\right\}=\frac{\Gamma((q+2) / s) r^{-(2 / s)}}{4 n(n+1) \Gamma(q / s)}(\boldsymbol{\Sigma} \otimes \boldsymbol{\Sigma}), \quad \forall_{j} \neq k \in\{1,2,3,4\}
$$


(ii)

$$
E_{\mathbf{X}}\left\{\operatorname{vec}\left(\mathbf{Y}_{i_{j}} \mathbf{Y}_{i_{k}}^{\prime}\right) \operatorname{vec}^{\prime}\left(\mathbf{Y}_{i_{k}} \mathbf{Y}_{i_{j}}^{\prime}\right)\right\}=\frac{\Gamma((q+2) / s) r^{-(2 / s)}}{4 n(n+1) \Gamma(q / s)}(\boldsymbol{\Sigma} \otimes \boldsymbol{\Sigma}) \mathbf{C}_{2}, \quad \forall_{j} \neq k \in\{1,2,3,4\}
$$

(iii)

$$
E_{\mathbf{X}}\left\{\operatorname{vec}\left(\mathbf{Y}_{j_{1}} \mathbf{Y}_{j_{2}}^{\prime}\right) \operatorname{vec}^{\prime}\left(\mathbf{Y}_{j_{3}} \mathbf{Y}_{j_{4}}^{\prime}\right)\right\}=\mathbf{0}_{4 \times 4}, \quad \forall\left(j_{1}, j_{2}, j_{3}, j_{4}\right) \in P\left(i_{1}, i_{1}, i_{2}, i_{3}\right)
$$

(iv)

$$
E_{\mathbf{X}}\left\{\operatorname{vec}\left(\mathbf{Y}_{j_{1}} \mathbf{Y}_{j_{2}}^{\prime}\right) \operatorname{vec}^{\prime}\left(\mathbf{Y}_{j_{3}} \mathbf{Y}_{j_{4}}^{\prime}\right)\right\}=\mathbf{0}_{4 \times 4}, \quad \forall\left(j_{1}, j_{2}, j_{3}, j_{4}\right) \in P\left(i_{1}, i_{1}, i_{1}, i_{2}\right)
$$

(v)

$$
E_{\mathbf{X}}\left\{\operatorname{vec}\left(\mathbf{Y}_{i_{1}} \mathbf{Y}_{i_{2}}\right) \operatorname{vec}^{\prime}\left(\mathbf{Y}_{i_{3}} \mathbf{Y}_{i_{4}}^{\prime}\right)\right\}=\mathbf{0}_{4 \times 4}
$$

where, for each $n \in \mathbb{N}, P\left(a_{1}, \ldots, a_{n}\right)$ denotes the group of permutations of $\left(a_{1}, \ldots, a_{n}\right)$.

Proof These properties are immediately deduced as a consequence of Lemma 1, taking into account that the matrices which appear in (i), (ii) and (iii) are submatrices of $\boldsymbol{\Xi}_{4}\left(\boldsymbol{\Sigma}^{1 / 2} \mathbf{Y}_{2}\right)$, the matrix in (iv) is a submatrix of $\Xi_{4}\left(\Sigma^{1 / 2} \mathbf{Y}_{3}\right)$ and the one in (v) is a submatrix of $\Xi_{4}\left(\Sigma^{1 / 2} \mathbf{Y}_{4}\right)$, being $\mathbf{Y}_{i}=U \mathbf{Z}_{i}$ with $U$ and $\mathbf{Z}_{i}$ independent and such that $U^{2} \sim \beta(i, n-i)$ and $\mathbf{Z}_{i} \sim K_{2 \times i}\left(\mathbf{0}, \mathbf{I}_{2} \otimes \mathbf{I}_{i}, q-i, r, s\right)$.

From this theorem, together with Lemmas 1 and 2, we have obtained the covariance matrix of $\hat{\boldsymbol{\Sigma}}^{*}$, being its determinant

$$
\begin{aligned}
\operatorname{det}\left(\operatorname{Cov}\left(\hat{\boldsymbol{\Sigma}}^{*}, \boldsymbol{\Sigma}\right)\right)= & \frac{4 n^{2} \Gamma(q / s)^{3} \Gamma((q+2) / s)^{3}}{\left(n^{2}-1\right)^{3} \Gamma((q+1) / s)^{6}} \\
& \times\left(n-\frac{\left(n^{2}-1\right) \Gamma((q+1) / s)^{2}}{n \Gamma(q / s) \Gamma(q+2) / s}\right)(\operatorname{det} \boldsymbol{\Sigma})^{3}, \quad q>1
\end{aligned}
$$

Then, for $q>2$, the efficiency equation associated with this estimator is given by

$$
\frac{4 \Gamma((q+1) / s)^{6}}{q s \Gamma(q / s)^{3} \Gamma((q+2) / s)^{3}}=\left(\frac{n^{2}+q s}{n+1}\right)^{2}\left(\frac{n}{n^{2}-1}\right)^{3}\left(n-\frac{\left(n^{2}-1\right) \Gamma((q+1) / s)^{2}}{n \Gamma(q / s) \Gamma(q+2) / s}\right)
$$

Although, there are some particular cases in which it is possible to find $q, s$ and $n$ solutions of this equation (being then $\hat{\boldsymbol{\Sigma}}^{*}$ an efficient estimator of $\boldsymbol{\Sigma}$ ), this does not happen in general.

For this reason, we have tried to search efficient estimators inside the family of unbiased linear estimators of $\boldsymbol{\Sigma}$, defined by

$$
\mathcal{F}_{\Sigma}^{K}=\left\{\hat{\boldsymbol{\Sigma}}=\frac{2 n^{2} \Gamma(q / s) r^{(1 / 2)}}{(n-1) \Gamma(q+1) / 2}\left(\sum_{i=1}^{n} \beta_{i}\right)^{-1} \sum_{i=1}^{n} \beta_{i}\left(\mathbf{X}_{i}-\overline{\mathbf{X}}\right)\left(\mathbf{X}_{i}-\overline{\mathbf{X}}\right)^{\prime} / \beta_{i} \in \mathbb{R}\right\}
$$


However, it is not possible to obtain such estimators. Actually, the efficiency equation associated with the estimators of this family is given by, for $q>2$,

$$
\begin{aligned}
k= & \left(\sum_{i=1}^{n} \beta_{i}\right)^{-6}\left[\sum_{i=1}^{n} \beta_{i}^{2}+\frac{1}{(n-1)^{2}} 2 \sum_{i=1}^{n} \sum_{\substack{j=1 \\
i<j}}^{n} \beta_{i} \beta_{j}\right]^{2} \\
& \times\left[\begin{array}{c}
\left(2-\frac{(n+1) \Gamma((q+1) / s)^{2}}{n \Gamma(q / s) \Gamma(q+2) / s}\right) \sum_{i=1}^{n} \beta_{i}^{2} \\
+\left(\frac{1+(n-1)^{2}}{(n-1)^{2}}-\frac{(n+1) \Gamma((q+1) / s)^{2}}{n \Gamma(q / s) \Gamma(q+2) / s}\right)^{2} \sum_{i=1}^{n} \sum_{\substack{j=1 \\
i<j}}^{n} \beta_{i} \beta_{j}
\end{array}\right],
\end{aligned}
$$

where

$$
k=\frac{4}{q s}\left(\frac{n+1}{n^{2}+q s}\right)^{2}\left(\frac{n+1}{n}\right)^{3} \frac{\Gamma((q+1) / s)^{6}}{\Gamma(q / s)^{3} \Gamma((q+2) / s)^{3}}
$$

and, if $\beta_{1}, \ldots, \beta_{n}$ would be solutions of this equation, they might verify the following property

$$
\min _{\beta_{1}, \ldots, \beta_{n}}\left(\sum_{i=1}^{n} \beta_{i}\right)^{-2} 2 \sum_{i=1}^{n} \sum_{\substack{j=1 \\ i<j}}^{n} \beta_{i} \beta_{j}=\frac{n-1}{n}
$$

As it is not possible to find $\beta_{1}, \ldots, \beta_{n}$ satisfying this condition (except for particular cases of $q, s, n)$, we conclude that there are not efficient linear estimators different from that indicated earlier.

Then, our aim is focused on determining the best unbiased linear estimators in the sense of minimizing the distance to the Cramér-Rao lower bound. For this purpose, the determinant of the covariance matrix of the estimators in this family is given by

$$
\begin{aligned}
\min \left\{\frac{\operatorname{det}(\operatorname{Cov}(\hat{\boldsymbol{\Sigma}}, \boldsymbol{\Sigma}))}{\hat{\boldsymbol{\Sigma}} \in \mathcal{F}_{\Sigma}^{K}}\right\}= & \frac{4 n^{3} \Gamma(q / s)^{3} \Gamma((q+2) / s)^{3}}{\left(n^{2}-1\right)^{3} \Gamma((q+1) / s)^{6}} \\
& \times\left(n-\frac{\left(n^{2}-1\right) \Gamma((q+1) / s)^{2}}{n \Gamma(q / s) \Gamma(q+2) / s}\right)(\operatorname{det} \boldsymbol{\Sigma})^{3}
\end{aligned}
$$

and, taking into account (17), we conclude that the sample covariance matrix, weighted by an unbiasedness constant, is an unbiased linear estimator of minimum distance to the Cramér-Rao lower bound.

\subsection{Estimators of $(\mu, \Sigma)$}

From the considerations made in the previous sections and the structure of the Fisher information matrix, the joint estimator, $\left(\mathbf{X}, \hat{\mathbf{\Sigma}}^{*}\right)$, is an unbiased linear estimator of minimum distance to the Cramér-Rao lower bound.

\section{Numerical simulation results}

The results theoretically obtained in the previous sections are illustrated in the numerical simulation example we next present. Specifically, our aim in this example is to show clearly 
Table 1. Unbiased linear estimations of $\boldsymbol{\mu}$ for different values of their weightings.

\begin{tabular}{|c|c|c|c|}
\hline Weightings, $\alpha_{i}$ & Estimations, $\hat{\boldsymbol{\mu}}$ & Weightings, $\alpha_{i}$ & Estimations, $\hat{\mu}$ \\
\hline $\begin{array}{l}\alpha_{2 i}=0.9 \\
\alpha_{2 i+1}=1\end{array}$ & $\hat{\mu}_{1}=\left(\begin{array}{c}-0.9467 \\
3.981\end{array}\right)$ & $\begin{array}{c}\alpha_{2 i}=1 \\
\alpha_{2 i+1}=2\end{array}$ & $\hat{\boldsymbol{\mu}}_{10}=\left(\begin{array}{c}-0.9342 \\
3.9342\end{array}\right.$ \\
\hline $\begin{array}{l}\alpha_{2 i}=0.8 \\
\alpha_{2 i+1}=1\end{array}$ & $\hat{\mu}_{2}=\left(\begin{array}{c}-0.9419 \\
3.963\end{array}\right)$ & $\begin{array}{c}\alpha_{2 i}=1 \\
\alpha_{2 i+1}=3\end{array}$ & $\hat{\mu}_{11}=\left(\begin{array}{c}-0.9404 \\
3.9576\end{array}\right.$ \\
\hline $\begin{array}{l}\alpha_{2 i}=0.7 \\
\alpha_{2 i+1}=1\end{array}$ & $\hat{\mu}_{3}=\left(\begin{array}{c}-0.9378 \\
3.9476\end{array}\right)$ & $\begin{array}{c}\alpha_{2 i}=1 \\
\alpha_{2 i+1}=4\end{array}$ & $\hat{\boldsymbol{\mu}}_{12}=\left(\begin{array}{c}-0.9442 \\
3.9726\end{array}\right.$ \\
\hline $\begin{array}{l}\alpha_{2 i}=0.6 \\
\alpha_{2 i+1}=1\end{array}$ & $\hat{\mu}_{4}=\left(\begin{array}{c}-0.9342 \\
3.9342\end{array}\right)$ & $\begin{array}{c}\alpha_{2 i}=1 \\
\alpha_{2 i+1}=5\end{array}$ & $\hat{\mu}_{13}=\left(\begin{array}{c}-0.9467 \\
3.981\end{array}\right.$ \\
\hline $\begin{array}{l}\alpha_{2 i}=0.5 \\
\alpha_{2 i+1}=1\end{array}$ & $\hat{\mu}_{5}=\left(\begin{array}{c}-0.931 \\
3.9225\end{array}\right)$ & $\begin{array}{c}\alpha_{2 i}=1 \\
\alpha_{2 i+1}=6\end{array}$ & $\hat{\boldsymbol{\mu}}_{14}=\left(\begin{array}{c}-0.9484 \\
3.9876\end{array}\right.$ \\
\hline $\begin{array}{l}\alpha_{2 i}=0.4 \\
\alpha_{2 i+1}=1\end{array}$ & $\hat{\mu}_{6}=\left(\begin{array}{c}-0.9283 \\
3.9122\end{array}\right)$ & $\begin{array}{c}\alpha_{2 i}=1 \\
\alpha_{2 i+1}=7\end{array}$ & $\hat{\boldsymbol{\mu}}_{15}=\left(\begin{array}{c}-0.9509 \\
3.9966\end{array}\right.$ \\
\hline $\begin{array}{l}\alpha_{2 i}=0.3 \\
\alpha_{2 i+1}=1\end{array}$ & $\hat{\mu}_{7}=\left(\begin{array}{c}-0.9259 \\
3.9031\end{array}\right)$ & $\begin{array}{c}\alpha_{2 i}=1 \\
\alpha_{2 i+1}=8\end{array}$ & $\hat{\mu}_{16}=\left(\begin{array}{c}-0.9498 \\
3.9926\end{array}\right.$ \\
\hline $\begin{array}{l}\alpha_{2 i}=0.2 \\
\alpha_{2 i+1}=1\end{array}$ & $\hat{\mu}_{8}=\left(\begin{array}{c}-0.9237 \\
3.8949\end{array}\right)$ & $\begin{array}{c}\alpha_{2 i}=1 \\
\alpha_{2 i+1}=9\end{array}$ & $\hat{\mu}_{17}=\left(\begin{array}{c}-0.9517 \\
3.9997\end{array}\right.$ \\
\hline $\begin{array}{l}\alpha_{2 i}=0.1 \\
\alpha_{2 i+1}=1\end{array}$ & $\hat{\mu}_{9}=\left(\begin{array}{c}-0.9217 \\
3.8875\end{array}\right)$ & $\begin{array}{c}\alpha_{2 i}=100 \\
\alpha_{2 i+1}=0.01\end{array}$ & $\hat{\mu}_{18}=\left(\begin{array}{c}-0.8743 \\
3.7621\end{array}\right.$ \\
\hline
\end{tabular}

Table 2. Unbiased linear estimations of $E$ for different values of their weightings.

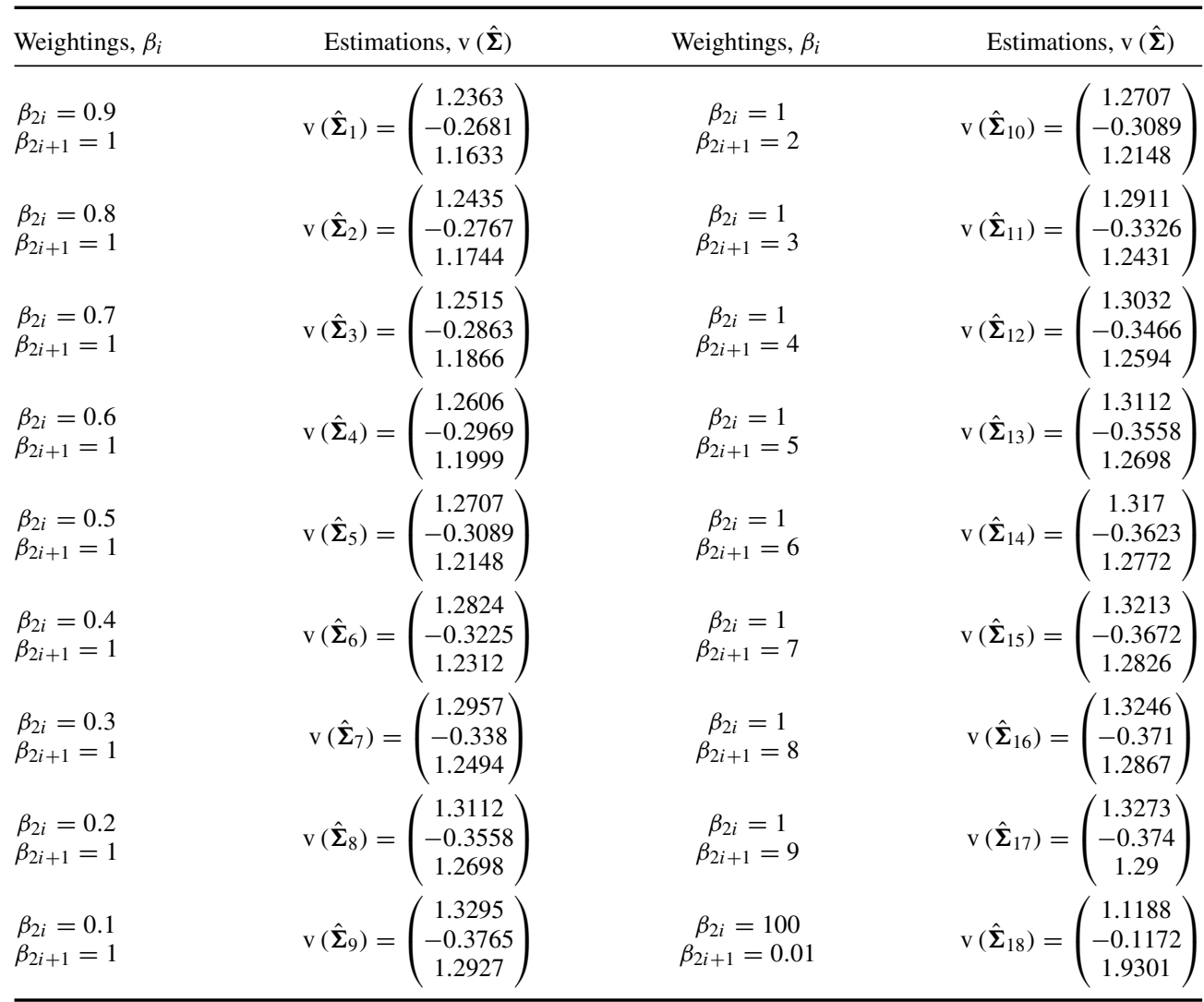


that the sample mean and the sample covariance matrix, affected by an unbiasedness weighting, minimize the distance to the Cramér-Rao lower bound.

By means of the method of simulating values of random matrices based on the knowledge of their matrix-variate conditional and marginal distributions, we have simulated, by using MATLAB program, a matrix value for a random matrix, $\mathbf{X}$, distributed as $K_{2 \times 100}\left((-1,4)^{\prime} \mathbf{1}^{\prime}, I_{2} \otimes I_{100}, 4,1,1\right)$. Next, from these simulations, by using expressions (16) and (18), we have obtained different unbiased linear estimations of $\boldsymbol{\mu}$ and $\boldsymbol{\Sigma}$, taking different values for $\left\{\alpha_{i}\right\}_{i=1, \ldots, 100}$ and $\left\{\beta_{i}\right\}_{i=1, \ldots, 100}$, respectively; specifically, these are the values for the sample mean and the sample covariance matrix

$$
\overline{\mathbf{x}}=\left(\begin{array}{c}
-0.9523 \\
4.0022
\end{array}\right), \quad \hat{\mathbf{\Sigma}}^{*}=\left(\begin{array}{cc}
1.2298 & -0.2603 \\
-0.2603 & 1.1531
\end{array}\right)
$$

and the remaining estimations for $\boldsymbol{\mu}$, and $\boldsymbol{\Sigma}$ are shown in tables 1 and 2, respectively. For all of them (including $\overline{\mathbf{x}}$ and $\hat{\mathbf{\Sigma}}^{*}$ ), we have also calculated the distances to the Cramér-Rao lower bound. The results obtained are presented in figures 1 and 2 .

From table 1 and figure 1, it is clearly observed that the smallest distance to the Cramér-Rao lower bound corresponds to the sample mean. Moreover, as $\alpha_{2 i}$ decreases taking $\alpha_{2 i+1}$ fixed and equal to 1 (this occurs from $\hat{\mu}_{1}$ to $\hat{\mu}_{9}$ ), the distances to the Cramér-Rao lower bound are worse. The same consideration can be made from $\hat{\mu}_{10}$ to $\hat{\mu}_{17}$ (obtained taking $\alpha_{2 i}$ fixed and equal to 1 and increasing $\alpha_{2 i+1}$ ). The worst estimation corresponds to $\hat{\mu}_{18}$, obtained taking a large value for $\alpha_{2 i}$ and a small value for $\alpha_{2 i+1}$.

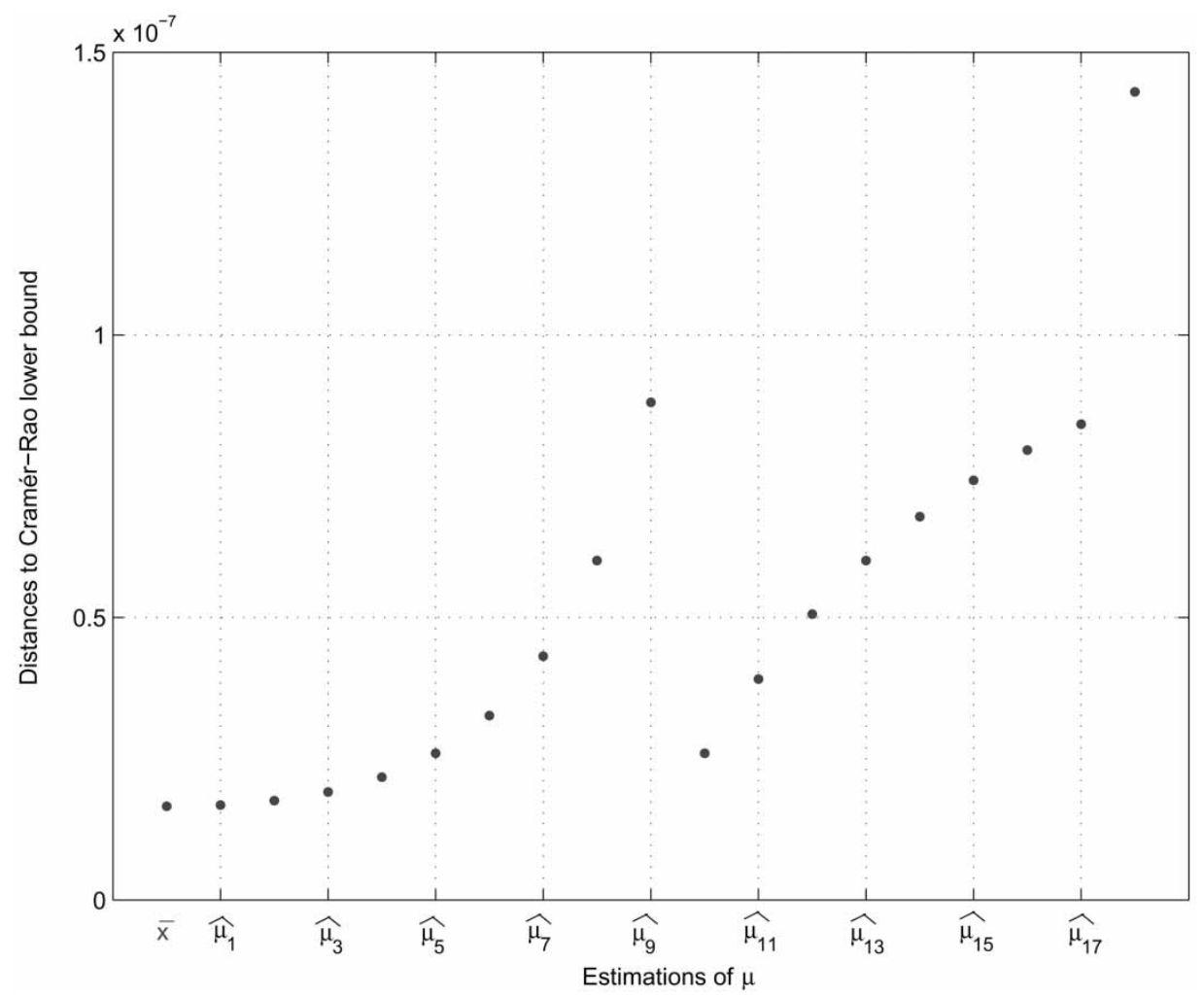

Figure 1. Distances to Cramér-Rao lower bound for the different unbiased linear estimations of $\mu$. 


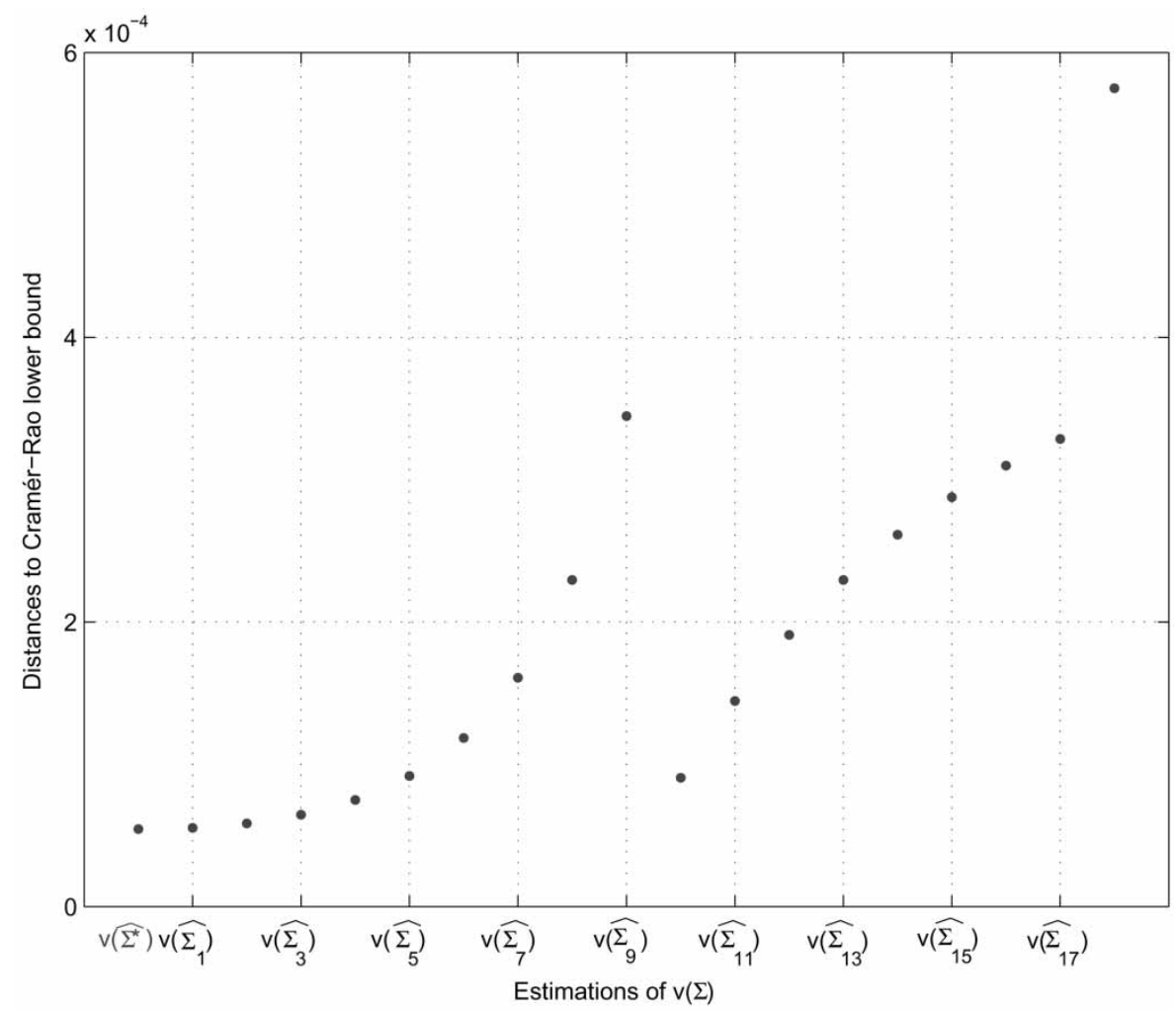

Figure 2. Distances to Cramér-Rao lower bound for the different unbiased linear estimations of $\boldsymbol{\Sigma}$.

Let us note that analogous considerations can be made from table 2 and figure 2 . Hence, we can conclude that the sample mean and the sample covariance matrix, affected by an unbiasedness weighting, are the best unbiased linear estimations of the parameters $\boldsymbol{\mu}$, and $\boldsymbol{\Sigma}$, respectively, in the sense of minimizing the distance to the Cramér-Rao lower bound.

\section{Conclusions}

This work approaches the search of efficient estimators of the parameters of the bivariate Kotz type distribution assuming a particular joint dependence between the sample random vectors.

Firstly, from the analogy with the normal law, it is reasonable to think that the sample mean and the sample covariance matrix, affected by an unbiasedness weighting, may be efficient estimators. As this consideration is not true, secondly we try to find such estimators inside the family of unbiased linear estimators of the parameters but we prove that there are not efficient linear estimators. Finally, our interest is focused in determining the best unbiased linear estimators in this family, obtaining that these estimators are the sample mean and the sample covariance matrix, weighted by an unbiasedness constant. From a numerical simulation study, it is shown that both estimators are the best in the sense of minimizing the distance to the Cramér-Rao lower bound. 


\section{Acknowledgements}

The authors would like to express their hearty gratitude to the Associate Editor and the anonymous referees for their invaluable suggestions in improving the original paper. This work has been partially supported by the 'Ministerio de Ciencia y Tecnología' under contracts BFM2002-03633 and BFM2002-00932.

\section{References}

[1] Fang, K.T. and Zhang, Y.T., 1990, Generalized Multivariate Analysis (New York: Springer-Verlag).

[2] Gupta, A.K. and Varga, T., 1992, Elliptically Contoured Models in Statistics (Dordrecht: Kluwer Academic Publishers).

[3] Shapiro, A. and Browne, M., 1987, Analysis of covariance structures under elliptical distributions. Journal of the American Statistical Association, 82(400), 1092-1097.

[4] Sutradhar, B.C., 1993, Score test for the covariance matrix of the elliptical $t$-distribution. Journal of Multivariate Analysis, 46, 1-12.

[5] Golan Kibria, B.M. and Safiul Haq, M., 1999, Predictive inference for the elliptical linear model. Journal of Multivariate Analysis, 68, 235-249.

[6] Chu, K., 1973, Estimation and decision for linear systems with elliptical random processes. IEEE Transactions on Automatic Control, 18, 499-505.

[7] Girón, F.J. and Rojano, J.C., 1994, Bayesian Kalman filtering with elliptically contoured errors. Biometrika, 81(2), 390-395.

[8] Gutiérrez, R. and Jiménez, J.D., 2001, Unbiased linear estimators of minimum distance in Pearson type II distribution. Proceedings of the IASTED International Conference on Applied Simulation and Modelling, Marbella, Spain, pp. 41-46.

[9] Gutiérrez, R. and Jiménez, J.D., 2002, On the efficiency property of the linear estimators in the bivariate Pearson type VII distribution. Journal of Statistical Computation and Simulation, 72(12), 937-948.

[10] Magnus, J.R. and Neudecker, H., 1988, Matrix Differential Calculus with Applications in Statistics and Econometrics (New York: John Wiley \& Sons).

[11] Muirhead, R.J., 1982, Aspects of Multivariate Statistical Theory (New York: John Wiley \& Sons). 